\title{
A hadronuclear interpretation of a high-energy neutrino event coincident with a blazar flare
}

\author{
Ruo-Yu Liu ${ }^{1}$, Kai Wang ${ }^{2,4}$, Rui Xue ${ }^{3}$, Andrew M. Taylor ${ }^{1}$, Xiang-Yu Wang ${ }^{3}$, Zhuo Li ${ }^{2,4}$, Huirong Yan ${ }^{1,5}$ \\ ${ }^{1}$ Deutsches Elektronen Synchrotron (DESY), Platanenallee 6, D-15738 Zeuthen, Germany; \\ ${ }^{2}$ Department of Astronomy, School of Physics, Peking University, Beijing 100871, China; \\ ${ }^{3}$ School of Astronomy and Space Science, Nanjing University, Nanjing, 210093, China; \\ ${ }^{4}$ Kavli Institute for Astronomy and Astrophysics, Peking University, Beijing 100871, China; \\ ${ }^{5}$ Institut für Physik und Astronomie, Universität Potsdam, D-14476 Potsdam, Germany
}

\begin{abstract}
Although many high-energy neutrinos detected by the IceCube telescope are believed to have an extraterrestrial origin, their astrophysical sources remain a mystery. Recently, an unprecedented discovery of a high-energy muon neutrino event coincident with a multiwavelength flare from a blazar, TXS $0506+056$, shed some light on the origin of the neutrinos. It is usually believed that a blazar is produced by a relativistic jet launched from an accreting supermassive black hole (SMBH). Here we show that the high-energy neutrino event can be interpreted by the inelastic hadronuclear interactions between the accelerated cosmic-ray protons in the relativistic jet and the dense gas clouds in the vicinity of the SMBH. Such a scenario only requires a moderate proton power in the jet, which could be much smaller than that required in the conventional hadronic model which instead calls upon the photomeson process. Meanwhile, the flux of the multiwavelength flare from the optical to gamma-ray band can be well explained by invoking a second radiation zone in the jet at a larger distance to the SMBH.
\end{abstract}

Detection of extraterrestrial high-energy neutrinos opens a new era of neutrino astronomy[1]. The approximate isotropic distribution of these neutrino events in the sky suggests a large fraction comes from extragalactic sources. It is commonly accepted that high-energy neutrinos are produced in the hadronic interactions of high-energy cosmic rays with matter or with photon fields inside the sources, in which charged pions are generated and give birth to neutrinos when they decay. Various extragalactic astrophysical objects, such as starburst galaxies (e.g., [2 [4]), tidal disruption events (e.g., [5 7]), active galactic nuclei (AGN) (e.g., [8-12]), have been investigated as the possible neutrino sources. Recently, IceCube detected a very-high-energy muon neutrino event on 22 September 2017 which was identified by the Extremely High Energy track event selection [13]. The energy of the neutrino event is estimated to be between $200 \mathrm{TeV}$ and $7.5 \mathrm{PeV}$ at $90 \%$ C.L. with the most probable energy to be $\sim 300 \mathrm{TeV}$, by assuming a power-law neutrino spectrum with an index of -2 [14]. Coincidently, The Fermi Large Area Telescope (Fermi-LAT) reported that a blazar, or more specifically, a BL Lac object TXS 0506+056 at redshift $z=0.3365$ [15] is located inside the event error region of $1^{\circ}$, with an increase of the $0.1-300 \mathrm{GeV}$ flux by a factor of 6 during 2018 September 15-27 compared to the 3FGL flux [16]. The follow-up observations on this object by various telescopes in various wavelengths also returned positive detections, including a significant detection by MAGIC telescopes at $>100 \mathrm{GeV}$ [17], X-ray emissions by Swift/XRT and NuSTAR [18], optical emissions by the ASAS-SN survey and various telescopes [19], as well as emission in radio band by VLA $[20]$. The chance coincidence of the high-energy neutrino event with the multiwavelength flare is disfavoured at the $3 \sigma$ level [14], suggesting the BL Lac object TXS $0505+056$ may be counterpart of the neutrino event.
BL Lac objects are regarded as a species of AGN in the unification schemes, with a relativistic jet pointing closely to the observer. The SMBH that supplies the jet is usually found to be surrounded by partially ionised high-density clouds emitting broad lines at a distance of $d_{\mathrm{BLR}}=0.001-0.1 \mathrm{pc}$ to the SMBH, and hence the region is also known as the broad line region (BLR). It is usually believed that the BLR reprocesses a fraction of the luminosity of the SMBH accretion disk into its own emission. If the launched jet extracts a lot of energy from the $\mathrm{SMBH}$, the disk emission is relatively weak in a picture of jet-disk symbiosis [21], leading to a low luminosity of the BLR. The nondetection of the BLR emission from TXS $0506+056$ then could be due to a low BLR luminosity outshone by the bright nonthermal emission from the jet. Thus, we can still assume the presence of high-density BLR clouds in the vicinity of the SMBH for TXS 0506+056. Actually, weak BLR emission has been discovered in other BL Lac objects such as in BL Lacertae, Mrk 421 and Mrk 501 [e.g. 22 24] with a luminosity of $10^{40}-10^{42} \mathrm{erg} \mathrm{s}^{-1}$ ). The BLR clouds orbit the SMBH and naturally provide targets for inelastic hadronuclear interactions or proton-proton (hereafter, $p p)$ collisions once they enter the jet [25, 26].

The atomic density of a single BLR cloud is $\sim 10^{9}-$ $10^{11} \mathrm{~cm}^{-3}$, with a size of the cloud $10^{13}-10^{14} \mathrm{~cm}$ [27, 28]. The typical number of individual BLR clouds is estimated to be $\sim 10^{7}$. There may also exist diffuse inter-cloud medium of lower-density in the BLR 27]. The typical mass of BLR in a typical AGN, estimated from line emissions, is about $M_{\mathrm{BLR}} \sim 1 M_{\odot}$, but there probably exists much more gas emitting less efficiently and hence the total mass of gas in the BLR may be much higher, up to $10^{3}-10^{4} M_{\odot}$ in the extreme case 29]. This provides a high gas column density $N_{\mathrm{H}}=10^{24}\left(M_{\mathrm{BLR}} / 10 M_{\odot}\right)\left(d_{\mathrm{BLR}} / 3 \times 10^{16} \mathrm{~cm}\right)^{-2} \mathrm{~cm}^{-2}$ for 


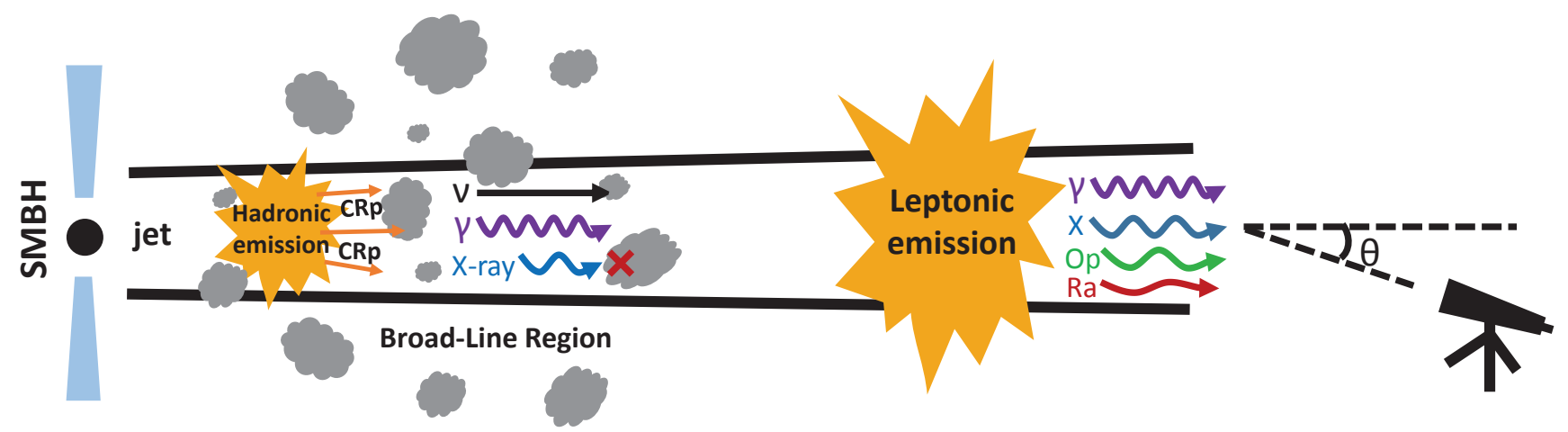

FIG. 1. A sketch illustration (not to scale) for our model. See text for more details.

neutrino production. Considering the possbility that heightened activity of the SMBH enhances the jet moving with an average bulk Lorentz factor $\Gamma_{j}$ (or a bulk velocity of $\left.v_{j}=c \sqrt{1-\Gamma_{j}^{-2}}\right)$. Some dissipation processes, such as internal collisions between different parts of the jet due to the velocity inhomogeneity, or via the internal-collision-induced magnetic reconnection and turbulence [30] mechanism, may occur inside or close to the BLR. If the jet loads a certain amount of protons, a fraction of the protons can be accelerated to relativistic energies and interact with gas in the BLR. To estimate the efficiency of the neutrino production in the system, we can compare the $p p$ collision timescale to the system dynamical timescale. To do this, we consider a mean atomic density in the BLR region by $n_{\mathrm{H}}=10^{8}\left(N_{\mathrm{H}} / 10^{24} \mathrm{~cm}^{-2}\right)\left(R_{\mathrm{BLR}} / 10^{16} \mathrm{~cm}\right)^{-1} \mathrm{~cm}^{-3}$ with $R_{\mathrm{BLR}}$ being the size of the BLR, and a $p p$ collision timescale in the jet frame can be given by $t_{p p}^{\prime} \simeq 8 \times$ $10^{5}\left(\Gamma_{j} / 20\right)^{-1}\left(n_{\mathrm{H}} / 10^{8} \mathrm{~cm}^{-3}\right)^{-1}\left(\sigma_{\mathrm{pp}} / 40 \mathrm{mb}\right)^{-1} \mathrm{~s}$ (hereafter primed quantities represent the quantities in the jet comoving frame), where $\sigma_{p p}$ is the cross section for the $p p$ collision. The system dynamical timescale is $t_{\mathrm{dyn}}^{\prime} \simeq$ $5 \times 10^{4}\left(d_{\mathrm{BLR}} / 3 \times 10^{16} \mathrm{~cm}\right)\left(\Gamma_{j} / 20\right)^{-1} \mathrm{~s}$. From this, one can see that due to the high gas density in the BLR, a proton can lose a considerable fraction of energy in the $p p$ collision. Protons may also escape the BLR by diffusive or advective transport. Generally, the limit of particle escape timescale can be estimated by $t_{\mathrm{esc}}^{\prime}=R_{\mathrm{BLR}} / c$ which is longer than the dynamical timescale $t_{\mathrm{dyn}}^{\prime}$. The particle escape process is therefore expected to have little influence on neutrino production rate. On the other hand, since the produced neutrino takes about $5 \%$ of the energy of the parent proton, to produce a neutrino of energy $E_{\nu}$, the proton energy in the jet frame needs to be $E_{p}^{\prime} \simeq 20 E_{\nu} / \Gamma_{j}=10^{15}\left(E_{\nu} / 10^{15}\right)\left(\Gamma_{j} / 20\right)^{-1} \mathrm{eV}$. The acceleration of a proton to this energy is required to be accomplished before the proton loses a significant fraction of their energies or within the dynamical timescale. Generally, the proton acceleration timescale can be estimated by $t_{\mathrm{acc}}^{\prime} \simeq 1000 \eta\left(E_{p}^{\prime} / 10^{15} \mathrm{eV}\right)\left(B_{j}^{\prime} / 0.1 \mathrm{G}\right)^{-1} \mathrm{~s}$, where $B_{j}^{\prime}$ is the magnetic field in the jet and $\eta \geq 1$ is a prefactor depending on the diffusion of CRs. We adopt $\eta=10$ in the following calculation. Comparing the acceleration timescale to the $p p$ cooling timescale $t_{p p}^{\prime}$ and the dynamical $t_{\mathrm{dyn}}^{\prime}$, we conclude that a proton is able to be accelerated well above $10^{15} \mathrm{eV}$ in the jet frame as long as the system is highly turbulent $(\eta \gtrsim 1)$. The injection spectrum of accelerated protons is assumed to follow a power-law distribution with an exponential cutoff, i.e., $\frac{d N_{p}^{\prime}}{d E_{p}^{\prime} d t^{\prime}} \propto E_{p}^{\prime-s} \exp \left(-E_{p}^{\prime} / E_{p, \max }^{\prime}\right)$ where $s$ is the spectral index and $E_{\max }^{\prime}$ is the maximum achievable proton energy determined by $t_{\mathrm{acc}}^{\prime}=\min \left(t_{\mathrm{dyn}}^{\prime}, t_{\mathrm{pp}}^{\prime}\right)$. Relevant timescales are shown in Supplementary Fig. 3,

High-energy electron/positron pairs and gamma-ray photons are also generated in the $p p$ collisions along with neutrinos. Unlike neutrinos, high-energy electrons/positrons and gamma-ray photons can initiate electromagnetic (EM) cascades in the BLR, by interacting with photon fields, magnetic fields and matter in the system via various mechanisms: for relativistic electron/positron, there are mainly three radiation processes, namely, synchrotron radiation in the magnetic field, inverse Compton radiation in the photon field and bremsstrahlung radiation in high-density gas, giving rise to multiwavelength emission; for gamma rays, the main interaction is the $\gamma \gamma$ annihilation with the background photon field in the BLR. An electron/positron pair will be generated in each $\gamma \gamma$ annihilation. For simplicity, we assume a homogeneous distribution of the photon density inside the BLR. The photon spectrum is assumed to be a grey body distribution with a dilution factor $c_{\mathrm{BLR}}$ which is obtained by $L_{\mathrm{BLR}} R_{\mathrm{BLR}} / c=c_{\mathrm{BLR}} a T_{\mathrm{BLR}}^{4} R_{\mathrm{BLR}}^{3}$. Here $a$ is the radiation density constant and the temperature $T_{\mathrm{BLR}}$ is assumed to be $22000 \mathrm{~K}$ so that after multiplying the Boltzmann constant $k$ we have $k T_{\mathrm{BLR}}=1.9 \mathrm{eV}$ which is the energy of the $\mathrm{H} \alpha$ emission line. The intrinsic BLR luminosity of an AGN is usually comparable or several times larger than its narrow line luminosity [31], while the latter one of TXS $0506+056$ is found to be a few times $10^{41} \mathrm{erg} \mathrm{s}^{-1}[15]$. For reference, we assume 
an intrinsic BLR luminosity $L_{\mathrm{BLR}} \sim 3 \times 10^{41} \mathrm{erg} \mathrm{s}^{-1}$, such that the photon number density in the BLR around the peak energy $\varepsilon_{p} \simeq 2.82 k T_{\mathrm{BLR}}=5.4 \mathrm{eV}$ of the spectrum is $n_{\mathrm{ph}} \simeq 10^{10}\left(L_{\mathrm{BLR}} / 3 \times\right.$ $\left.10^{41} \mathrm{ergs}^{-1}\right)\left(R_{\mathrm{BLR}} / 10^{16} \mathrm{~cm}\right)^{-2}\left(k T_{\mathrm{BLR}} / 1.9 \mathrm{eV}\right)^{-1} \mathrm{~cm}^{-3}$. Gamma-ray photons around $100 \mathrm{GeV}$ will be absorbed by the photon field of the BLR, with an optical depth $\tau_{\gamma \gamma} \simeq n_{\mathrm{BLR}} \sigma_{\gamma \gamma} R_{\mathrm{BLR}} \simeq 10$ where $\sigma_{\gamma \gamma} \simeq 10^{-25} \mathrm{~cm}^{2}$ is approximately the peak cross section of the $\gamma \gamma$ annihilation. The typical energy of electrons/positrons generated by $100 \mathrm{GeV}$ photons is $50 \mathrm{GeV}$. These electrons/positrons will subsequently radiate $\sim 10 \mathrm{GeV}$ photons via inverse Compton scattering off the grey body radiation from the BLR with typical energies of a few $\mathrm{eV}$. As a result, the $10 \mathrm{GeV}$ gamma-ray flux will be enhanced. Note that the interaction rate of the photomeson process is roughly three orders of magnitude smaller than that of the $\gamma \gamma$ annihilation with the same target photon field [32], the photomeson process is henceforth negligible given a $\gamma \gamma$ annihilation opacity of only $\simeq 10$. Another thing worth noting is that there may also exist a dusty torus extending to a distance of $\sim 0.1-10 \mathrm{pc}$ from the SMBH, supplying an infrared photon field. In order not to introduce too many free parameters in our model, we do not consider the inclusion of infrared radiation field, but we show in the Supplement that including them is not expected to change our result.

We obtain the emission rate of secondary gammaray photons, electrons/positrons, and neutrinos with the semianalytic method developed by [33]. The EM cascades initiated by high-energy photons and electrons/positrons are calculated following the treatment in 34, 35, also see Supplement for details]. BLR clouds that enter the jet will be fully ionised by the UV/X-ray emission from the cascade electrons. Due to the high column density of BLR we consider, the ionised electrons will provide a large opacity for optical to X-ray photons by Compton scattering, while the gamma-ray photons escape due to the suppressed cross section (i.e., Klein-Nishina effect). Note that a large optical depth for $\mathrm{X}$-ray in the BLR is also needed in order not to overshoot the observed flux. To explain the multiwavelength emission, we invoke a second radiation zone beyond the BLR where the kinetic energy of the jet is dissipated, such as a dissipating "blob" which is usually employed to explain the multiwavelength emission of BL Lac objects in many previous models [e.g. 36 38]. We ascribe the multiwavelength emission to the synchrotron radiation and synchrotron self-Compton (SSC) of the nonthermal electrons accelerated in the blob. Note that having two (or more) radiation zones may not be unnatural. For example, if the dissipations are produced by internal collisions due to inhomogeneity in the jet speed, multiple collisions can occur at different places and form multiple radiation zones. Note that the velocity of blob outside the BLR may be smaller (but still relativistic) since some cloud material may be loaded in the jet when the jet pass through the BLR (see Supplement for discussion).
The key difference between the dissipation in the BLR and outside the BLR is the environment in which the dissipation takes place. If the dissipation does not take place inside or close to the BLR, then there would be too few target gas in the dissipation region for efficient $p p$ collision and subsequently little neutrino will be produced. Due to this reason, the neutrino emission is not necessarily expected to be temporally associated with the low-energy emission.

We consider two slopes for the accelerated proton spectrum in the BLR, say, $s=1.6$ and $s=2.0$. The predicted multiwavelength flux and neutrino flux are shown in Fig. 2. Model parameters are given in Supplementary Table. 1. We do not optimize the fitting (e.g., minimize the $\chi^{2}$ ) noting instead simply that a reasonable reduced $\chi^{2}$ is obtained. In the $s=1.6$ case, the optical flux are dominated by leptonic emission of the blob, X-ray and $\mathrm{TeV}$ emission are dominated by the hadronic emission in the BLR, while both the leptonic emission and the hadronic emission contribute to the $(0.1-100) \mathrm{GeV}$ flux. Differently, X-ray emission is dominated by the leptonic emission (SSC of electrons) in the $s=2.0$ case. This is because that, compared to the $s=2.0$ case, a larger amount of X-ray emitting electrons is generated in the BLR for $s=1.6$ through the EM cascade, given a harder spectrum and a larger proton power in the jet. As a consequence, the X-ray flux is still high after being scattered by nonrelativistic electrons in the BLR. Thus, in the $s=1.6$ case, we expect the temporal behaviour of $\mathrm{X}$-ray emission to be similar to that of the $\mathrm{TeV} /$ neutrino emission while in the $s=2.0$ case, X-ray temporal behaviour is similar to that of the optical emission. The radio flux can not be fitted in both cases due to the synchrotron self-absorption by the accelerated electrons. The difficulty of fitting the radio emission has been also found in other BL Lac objects with it being suggested that the radio emission arise from an extended region with a weaker magnetic field (e.g., 39]). One interesting feature in our model is that the superposition of the SSC emission in the blob and the hadronic emission in the BLR can reproduce the flat spectrum in $0.1-10 \mathrm{GeV}$ as observed by Fermi-LAT, while a pure leptonic model leads to a curved spectral shape, as is shown in the inset of Fig. 2, Considering that the effective area of IceCube for $200 \mathrm{TeV}$ neutrinos is of the order of $10 \mathrm{~m}^{2}$ in the direction of TXS $0506+056$ and is roughly proportional to the neutrino energy, we find that IceCube is expected to detect one muon (or anti-muon) neutrino event in $0.2-7.5 \mathrm{PeV}$ in 75 days for $s=1.6$ and in 3.2 years for $s=2.0$, should the SMBH activity lasts such a long period of time.

In this work, we propose a hadronuclear origin of the high-energy event from the BL object TXS 0506+056. The multiwavelength flare coincident with the neutrino event can also be explained under the same framework. Different from the conventional hadronic model for neutrino production in blazars which considers photomeson process [40 43], we ascribe the neutrino production to 


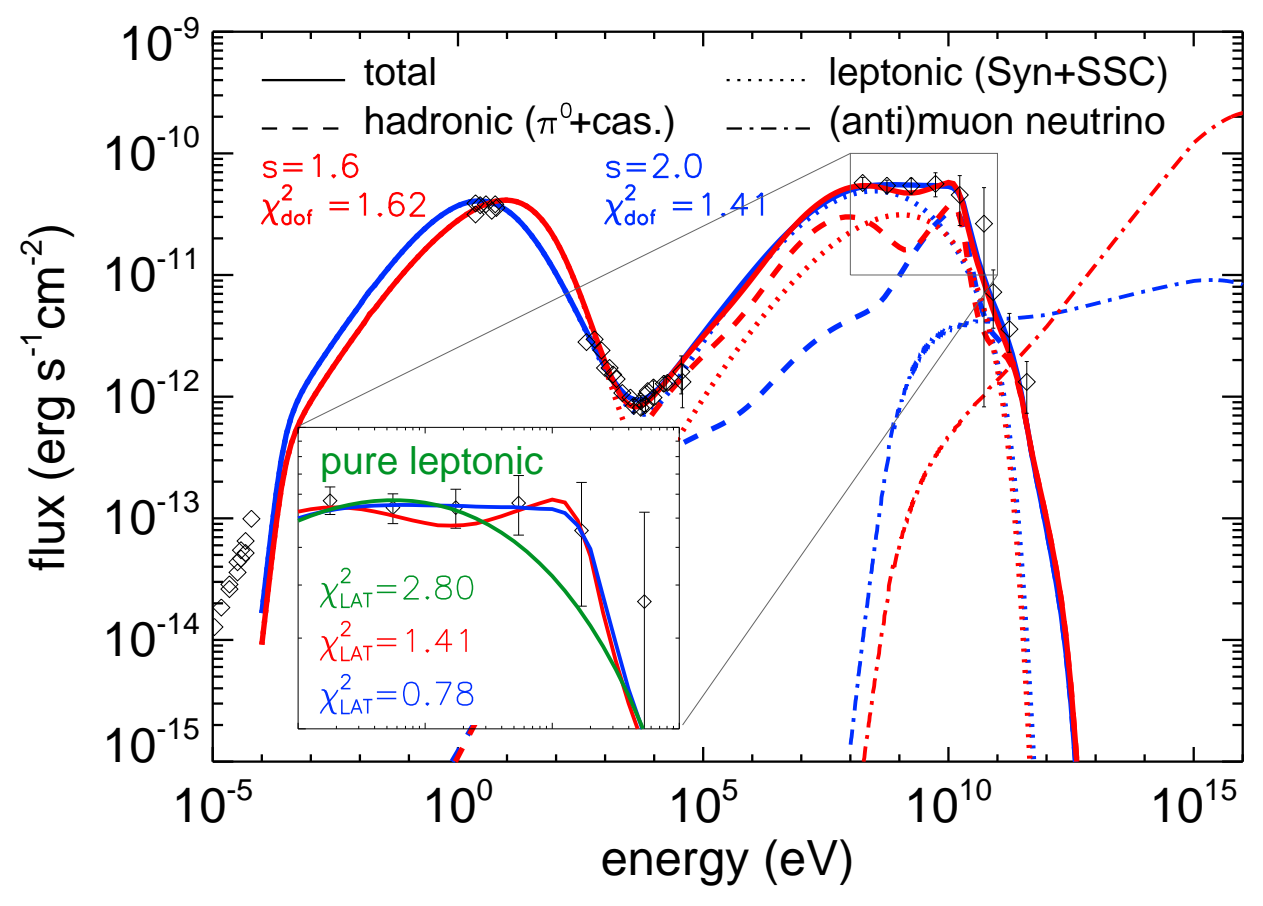

FIG. 2. Predicted multiwavelength flux and neutrino flux of TXS 0506+056 for $s=1.6$ (red) and $s=2.0$ (blue). The dotted and dashed curves are the flux from leptonic (including synchrotron and SSC radiation) and hadronic processes (including pionic emission and EM cascades). The dash-dotted curves represent the flux of $\nu_{\mu}+\bar{\nu}_{\mu}$ flux assuming a flavor ration of 1 : 1 : 1 after oscillation. The black diamonds are data points taken from [14]. To obtain a reasonable reduced chi-square value, we introduce an error of $2 \%$ for each optical data point, which is the typical level of the systematic error. The inset compares the fitting to the Fermi-LAT data by a pure leptonic model (green) and our model with $s=1.6$ and $s=2.0$.

the $p p$ collision by assuming a high column density gas in the BLR. Thus, the efficiency of the hadronic interaction can approach unity without introducing too large an internal $\gamma \gamma$ annihilation opacity for gamma rays. As a consequence, our model results in a moderate proton power of the jet, i.e., $L_{p} \sim 10^{45}-10^{46} \mathrm{erg} \mathrm{s}^{-1}$, which is about $(3-20) \%$ of the Eddington luminosity of a SMBH with a mass of $10^{9} M_{\odot}$. By contrast, the photomeson model usually leads to a quite low efficiency for neutrino production in order to avoid a large internal $\gamma \gamma$ annihilation opacity for gamma rays and hence has to invoke a huge proton luminosity that is close to or even far exceeds the Eddington luminosity of the SMBH. Thus, the $p p$ collision model alleviates the extreme requirement for the proton budget of the jet. The neutrino spectrum in the $p p$ collision scenario can extend down to GeV energy roughly following the proton spectrum, so in principle we may expect the detection of $<100 \mathrm{TeV}$ neutrino from TXS 0506+056 (perhaps implied by the earlier neutrino flare from this source [44]). What's more, our model predicts a high TeV-PeV gamma-ray flux at source, which will be reprocessed into diffuse $\mathrm{GeV}$ gamma rays through the EM cascades on the cosmic microwave background (CMB) and extragalactic background light (EBL). The potential of our model to explain multiwavelength radi- ation of other BL Lac objects will be studied and the results can be used to forecast their contributions to the diffuse gamma-ray background and the diffuse high-energy neutrino background.

\section{ACKNOWLEDGMENTS}

We thank Kohta Murase for valuable comments.This work is partially supported by 973 program grant $2014 \mathrm{CB} 845800$ and the NSFC grant 11625312 and 11851304 . 


\section{Supplement}

Hadronic emission in the BLR (including the cascade emission):

Denote the total luminosity of nonthermal protons (i.e., the beamed power of accelerated protons) in the BLR in the source frame by $L_{p, \mathrm{BLR}}$, we find the power of injected protons in the jet comoving frame to be $L_{p, \mathrm{BLR}} / \Gamma_{j}^{2}$. Assuming the differential proton spectrum at injection to be $\dot{N}_{p}^{\prime} \propto E_{p}^{\prime-s} \exp \left(-E_{p, \max }^{\prime}\right)$ in the jet comoving frame, we have $\int^{E_{p, \max }^{\prime}} E_{p}^{\prime} \dot{N}_{p}^{\prime} d E_{p}^{\prime}=L_{p, \mathrm{BLR}} / \Gamma_{j}^{2}$. The proton spectrum in the BLR can then be estimated by $N_{p}^{\prime}=\dot{N}_{p}^{\prime} t_{p}^{\prime}$ where $t_{p}^{\prime}=\min \left(t_{p p}^{\prime}, t_{\mathrm{dyn}}^{\prime}, t_{\mathrm{esc}}^{\prime}\right)$ with $t_{\mathrm{pp}}^{\prime}$ and $t_{\mathrm{dyn}}^{\prime}$ being the cooling time of protons due to the inelastic $p p$ collision, the diffuse escape timescale and the dynamical timescale in the jet comoving frame, respectively (see Fig. 3). The diffusive escape timescale of a proton from the BLR can be given by $t_{\mathrm{esc}}^{\prime}=R_{\mathrm{BLR}}^{2} / D \simeq$ $3 \times 10^{8} \eta^{-1}\left(R_{\mathrm{BLR}} / 10^{16} \mathrm{~cm}\right)^{2}\left(E_{p}^{\prime} / 10^{15} \mathrm{eV}\right)^{-1}\left(B_{j}^{\prime} / 0.1 \mathrm{G}\right)$ s where $D \simeq 3 \times 10^{23} \eta\left(E_{p}^{\prime} / 10^{15} \mathrm{eV}\right)\left(B_{j}^{\prime} / 0.1 \mathrm{G}\right)^{-1} \mathrm{~cm}^{2} \mathrm{~s}^{-1}$ is the diffusion coefficient. The results in the main text and Supplement are based on $\eta=10$. Note that the obtained escape timescale may be subject to large uncertainties since the escape of proton can be quite complex, which depends on a detailed specification of the geometry, the boundary conditions, and the local turbulence property. However, the limit of the escape timescale is roughly $R_{\mathrm{BLR}} / c$ (i.e., ballistic escape), and is always longer than the dynamical timescale. Thus, the uncertainty on the escape timescale will not have significant influence on our results.

Hadronuclear interactions between accelerated protons and atoms of in the BLR clouds produce neutral and charged pions, which eventually decay into gamma-ray photons, electrons/positrons, and neutrinos, i.e.,

$$
\begin{aligned}
& p+p \rightarrow \pi^{0} \rightarrow \gamma+\gamma \\
& p+p \rightarrow \pi^{+} \rightarrow \nu_{\mu}+\mu^{+} \rightarrow \nu_{\mu}+e^{+}+\nu_{e}+\bar{\nu}_{\mu} \\
& p+p \rightarrow \pi^{-} \rightarrow \bar{\nu}_{\mu}+\mu^{-} \rightarrow \bar{\nu}_{\mu}+e^{-}+\bar{\nu}_{e}+\nu_{\mu}
\end{aligned}
$$

The differential spectrum of the secondary particles produced in unit time are calculated following the method developed by [33] (see also [45, 46]), i.e.,

$$
\dot{N}_{i}^{\prime}\left(E_{i}^{\prime}\right) \equiv \frac{d N_{i}^{\prime}}{d E_{i}^{\prime} d t^{\prime}}=c n_{\mathrm{H}}^{\prime} \int_{E_{i}}^{\infty} \sigma_{p p} N_{p}^{\prime}\left(E_{p}^{\prime}\right) F_{i}\left(\frac{E_{i}^{\prime}}{E_{p}^{\prime}}, E_{p}^{\prime}\right) \frac{d E_{p}^{\prime}}{E_{p}^{\prime}}
$$

where $i$ could be $\gamma, e$ or $\nu$, and $F_{i}$ is the spectrum of the secondary $\gamma, e^{ \pm}$or $\nu$ in a single collision. This description works for $E_{p} \gtrsim 100 \mathrm{GeV}$, while for $E_{p}<100 \mathrm{GeV}$ a $\delta$-functional approximation for the energy of produced pions can be used to obtain the secondary spectrum

$$
\begin{aligned}
\dot{N}_{i}^{\prime}\left(E_{i}^{\prime}\right) & =2 c n_{\mathrm{H}}^{\prime} \frac{\tilde{n}}{K_{\pi}} \int_{E_{i, \min }^{\prime}}^{\infty} \sigma_{p p}\left(m_{p}+\frac{E_{\pi}^{\prime}}{K_{\pi}}\right) \\
& \times \chi_{i} \frac{d N_{p}}{d E_{p}}\left(m_{p}+\frac{E_{\pi}^{\prime}}{K_{\pi}}\right) \frac{d E_{\pi}^{\prime}}{\sqrt{E_{\pi}^{\prime 2}-m_{\pi}^{2}}}
\end{aligned}
$$

where $E_{\pi}^{\prime}$ is the energy of pions and the pion rest mass $m_{\pi} \simeq 135 \mathrm{MeV}$ for gamma-ray production and $m_{\pi} \simeq 140 \mathrm{MeV}$ for neutrino production. $E_{i, \text { min }}^{\prime}=E_{i}^{\prime} / \zeta_{i}+\zeta_{i} m_{\pi}^{2} / 4 E_{i}^{\prime}$, with $\zeta_{\gamma}=1$ for gamma rays, $\zeta_{e}=1$ for $e^{ \pm}$, (anti-)muon neutrinos and (anti-)electron neutrinos from $\mu^{ \pm}$decay), and $\zeta_{\nu}=1-m_{\mu}^{2} / m_{\pi}^{2}=0.427$ for (anti-)muon neutrino from $\pi^{ \pm}$decay. $m_{\mu} \simeq 106 \mathrm{MeV}$ is the muon rest mass), $\xi_{\gamma}=1, \xi_{\mu}=1$, and $\xi_{e}=\frac{35}{16}\left[1-\left(\frac{E_{e}^{\prime}}{E_{e, \max }^{\prime}}\right)^{2}\right]^{3}$ where $E_{e, \max }^{\prime}=\left(E_{\pi}^{\prime}+\sqrt{E_{\pi}^{\prime 2}-m_{\pi}^{2}}\right) / 2 . K_{\pi}=0.17$, and $\tilde{n}$ is a free parameter that is determined by the continuity of the flux of the secondary particle at $100 \mathrm{GeV}$.

The high-energy photons and electrons/positrons (hereafter we do not distinguish positrons from electrons) produced in $p p$ collisions will initiate EM cascades in the BLR via the synchrotron radiation, the inverse Compton (IC) scattering and $\gamma \gamma$ annihilation. As we can see in Fig. 3. the timescales of these cooling processes are shorter than the dynamical timescale, so we follow the treatment in Böttcher et al. 34], Wang et al. 35] for fast-cooling electrons which are assumed to be in quasi-steady state. Assuming a homogeneous spatial distribution of electrons in the BLR, the cascade equation for electrons is given by

$$
\frac{\partial N_{e}^{\prime}}{\partial t^{\prime}}+\frac{\partial}{\partial \gamma_{e}^{\prime}}\left(\dot{\gamma}_{e}^{\prime} N_{e}^{\prime}\right)=Q_{e, \pi}^{\prime}+Q_{e, \gamma \gamma}^{\prime}-\frac{N_{e}^{\prime}}{t_{e, \text { esc }}^{\prime}}
$$

where

$$
\dot{\gamma}_{e}^{\prime}=-\frac{4 c \sigma_{T}}{3 m_{e} c^{2}}\left(\frac{B_{j}^{\prime 2}}{8 \pi}+\Gamma_{j}^{2} c_{\mathrm{BLR}} a T_{\mathrm{BLR}}^{4} \kappa_{\mathrm{KN}}\left(\gamma_{e}^{\prime}\right)\right) \gamma_{e}^{\prime 2}
$$




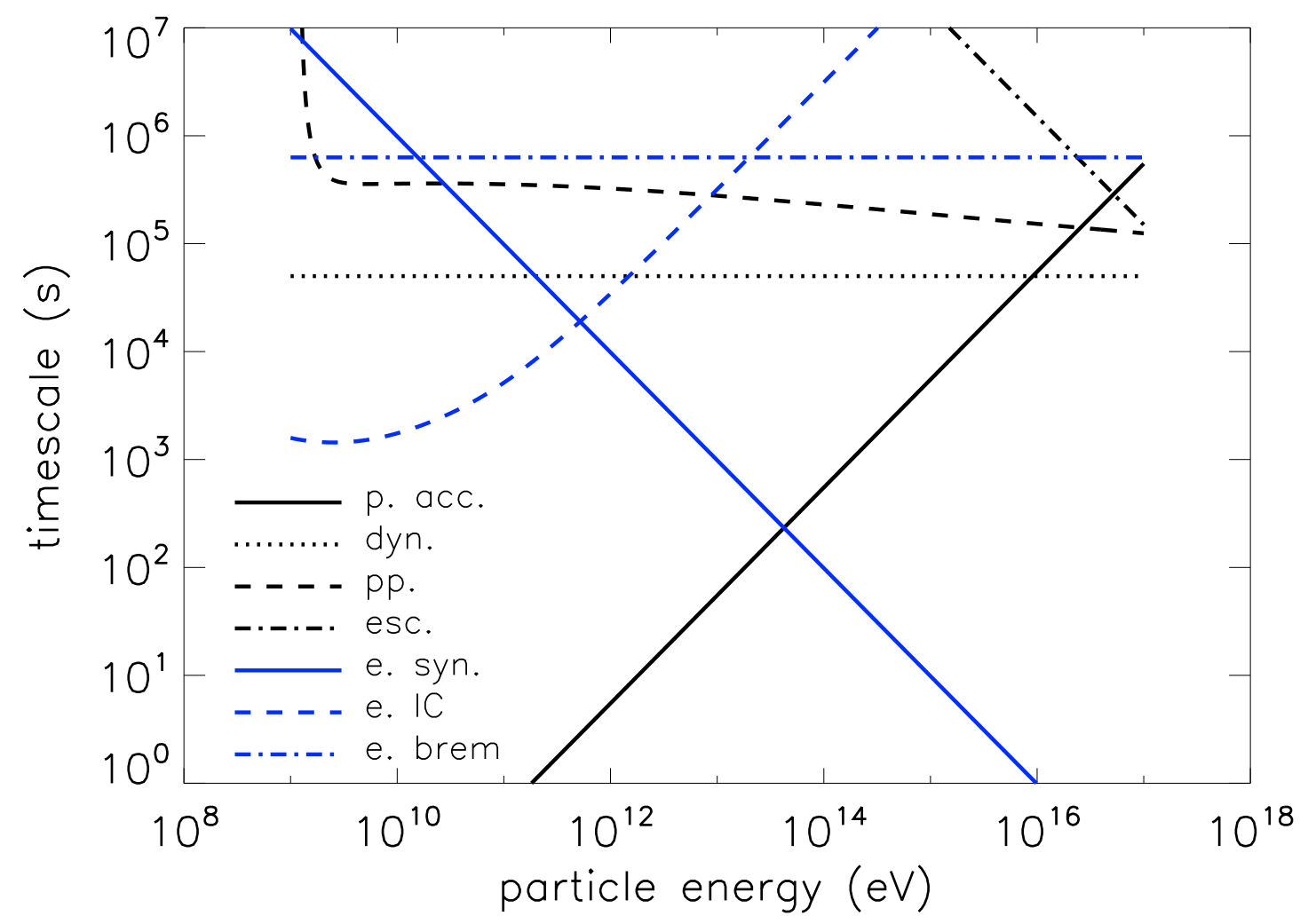

FIG. 3. Timescales of various processes in the BLR measured in the jet comoving frame. Parameters are the same with the ones shown in Table. [ for $s=2.0$.

is the energy loss rate of electrons due to the synchrotron radiation in the magnetic field of the jet and due to the IC radiation in the relativistic boosted photon field of the BLR. In the above equation, $\sigma_{T}$ is the Thomson cross section, $\kappa_{\mathrm{KN}}$ is a numerical factor considering modification of the Klein-Nishina effect to the energy loss rate. We here neglect the electron cooling due to bremsstrahlung radiation, since the cooling time of this process [47] $t_{\text {brem }}=6.3 \times 10^{5}\left(n_{\mathrm{H}}^{\prime} / 2 \times 10^{9} \mathrm{~cm}^{-3}\right)^{-1} \mathrm{~s}$ is much longer than the synchrotron or IC cooling timescale. $t_{e, \text { esc }}^{\prime}$ is the escape timescale of electrons from the BLR (or the residence timescale in the BLR), which is equal to the smaller one between the diffusion time $t_{e, \mathrm{diff}}=R_{\mathrm{BLR}}^{2} / D$ (the same with that for proton of the same energy) and the dynamic timescale $t_{\mathrm{dyn}}^{\prime}$. On the right-hand side of the equation, $Q_{e, \pi}^{\prime}=\dot{N}_{e}^{\prime}$ represents the injection of electrons from the $p p$ collision via pion decay and $Q_{e, \gamma \gamma}^{\prime}$ is the injection rate of electrons from $\gamma \gamma$ annihilation of gamma-ray photons, including the annihilation of the high-energy photons from the neutral pion decay produced in the $p p$ collision, and the high-energy photons produced by the synchrotron and the IC radiation, i.e.,

$$
\begin{aligned}
Q_{e, \gamma \gamma}\left(\gamma_{e}^{\prime}\right)^{\prime} & =f_{\mathrm{abs}}\left(E_{\gamma, 1}^{\prime}\right)\left(\dot{n}_{E_{\gamma, 1}^{\prime}}^{0}+\dot{n}_{E_{\gamma, 1}^{\prime}}^{\mathrm{sy}}+\dot{n}_{E_{\gamma, 1}^{\prime}}^{\mathrm{IC}}\right) \\
& +f_{\mathrm{abs}}\left(E_{\gamma, 2}^{\prime}\right)\left(\dot{n}_{E_{\gamma, 2}^{\prime}}^{0}+\dot{n}_{E_{\gamma, 2}^{\prime}}^{\mathrm{sy}}+\dot{n}_{E_{\gamma, 2}^{\prime}}^{\mathrm{IC}}\right),
\end{aligned}
$$

with

$$
f_{\mathrm{abs}}\left(E_{\gamma}^{\prime}\right)=1-\frac{1-e^{-\tau_{\gamma \gamma}\left(E_{\gamma}^{\prime}\right)}}{\tau_{\gamma \gamma}\left(E_{\gamma}^{\prime}\right)}
$$

being the absorbed fraction of photons. $\tau_{\gamma \gamma}$ is the optical depth of the high-energy photon of energy $E_{\gamma}^{\prime}$ due to $\gamma \gamma$ annihilation. Since the optical depth is a Lorentz invariant, we calculate it in the source frame by

$$
\tau_{\gamma \gamma}\left(E_{\gamma}^{\prime}\right)=\frac{2 R_{\mathrm{BLR}}}{E_{\gamma}} \int_{1}^{\infty} s \sigma_{\gamma \gamma}(s) \int_{s m_{e}^{2} c^{4} / 2 E_{\gamma}}^{\infty} \frac{n_{\mathrm{ph}}(\varepsilon)}{\varepsilon^{2}} d \varepsilon
$$


where $E_{\gamma}=\Gamma_{j} E_{\gamma}^{\prime}, \sqrt{s}$ is the center-of-momentum Lorentz factor of the produced pair, $\varepsilon$ is the photon energy of the BLR and $\sigma_{\gamma \gamma}$ is the total cross section for the $\gamma \gamma$ annihilation given by [48].

Two electrons are produced in each $\gamma \gamma$ annihilation, taking a fraction of $f_{\gamma}$ and $1-f_{\gamma}$ of the energy of the incident gamma-ray photon, respectively. Therefore, to the produce an electron with energy $\gamma_{e}^{\prime}$, the photons need to have the energy of either $E_{\gamma, 1}^{\prime}=\gamma_{e}^{\prime} / f_{\gamma}$, or $E_{\gamma, 2}^{\prime}=\gamma_{e}^{\prime} /\left(1-f_{\gamma}\right)$. That is the reason why Eq. (5) contains two parts. According to Böttcher et al. [34], taking $f_{\gamma}=0.9$ can lead to a cascade spectrum in a good agreement with the numerical Monte Carlo simulations.

In the quasi-steady state, we have $\frac{\partial N_{e}^{\prime}}{\partial t}=0$ and the solution to Eq. 3 is given by

$$
N_{e}^{\prime}\left(\gamma_{e}^{\prime}\right)=-\frac{1}{\dot{\gamma}_{e}^{\prime}} \int_{\gamma_{e}^{\prime}}^{\infty} d \tilde{\gamma}_{e}^{\prime}\left[Q_{e}\left(\tilde{\gamma}_{e}^{\prime}\right)+\dot{N}_{e, \gamma \gamma}^{\prime}\left(\tilde{\gamma}_{e}^{\prime}\right)-\frac{N_{e}^{\prime}\left(\tilde{\gamma}_{e}^{\prime}\right)}{t_{e, \text { esc }}^{\prime}}\right]
$$

Since the electron spectrum $N^{\prime}{ }_{e}\left(\gamma_{e}^{\prime}\right)$ appears at both sides of the Eq. (8), the electron spectrum is calculated progressively, namely, starting from the highest electron energies and then using the solution of $N_{e}^{\prime}\left(\gamma_{e}^{\prime}\right)$ for large $\gamma_{e}^{\prime}$ as one progress toward the lower values of $\gamma_{e}^{\prime}$, to obtain the final electron spectrum in the quasi-steady state. The obtained electron energy spectrum in the jet comoving frame is shown in Fig. 4. Then, we use the obtained $N_{e}^{\prime}$ to get the synchrotron and IC radiation of cascaded electrons in the quasi-steady state. In Fig. 5 , we decompose the hadronic emission in the BLR into difference components.

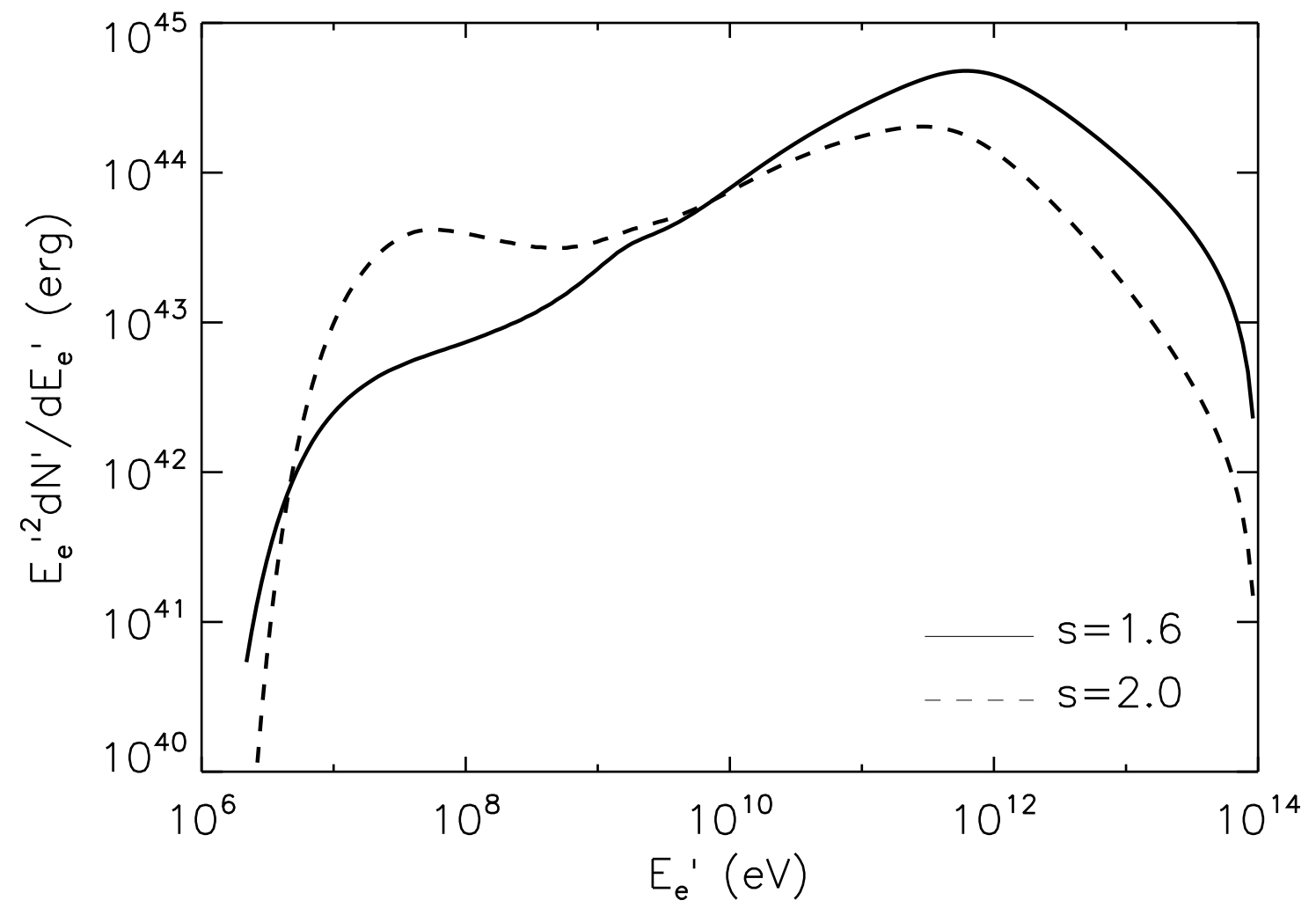

FIG. 4. Steady-state electron energy spectrum in the cascade. The solid and the dashed curves are for $s=1.6$ and $s=2.0$ respectively.

Ionisation of BLR clouds and Thomson optical depth for UV/X-ray photons emitted in the hadronic process:

The electrons generated in the EM cascade radiate strong UV/X-ray photons which can ionise the BLR clouds. 

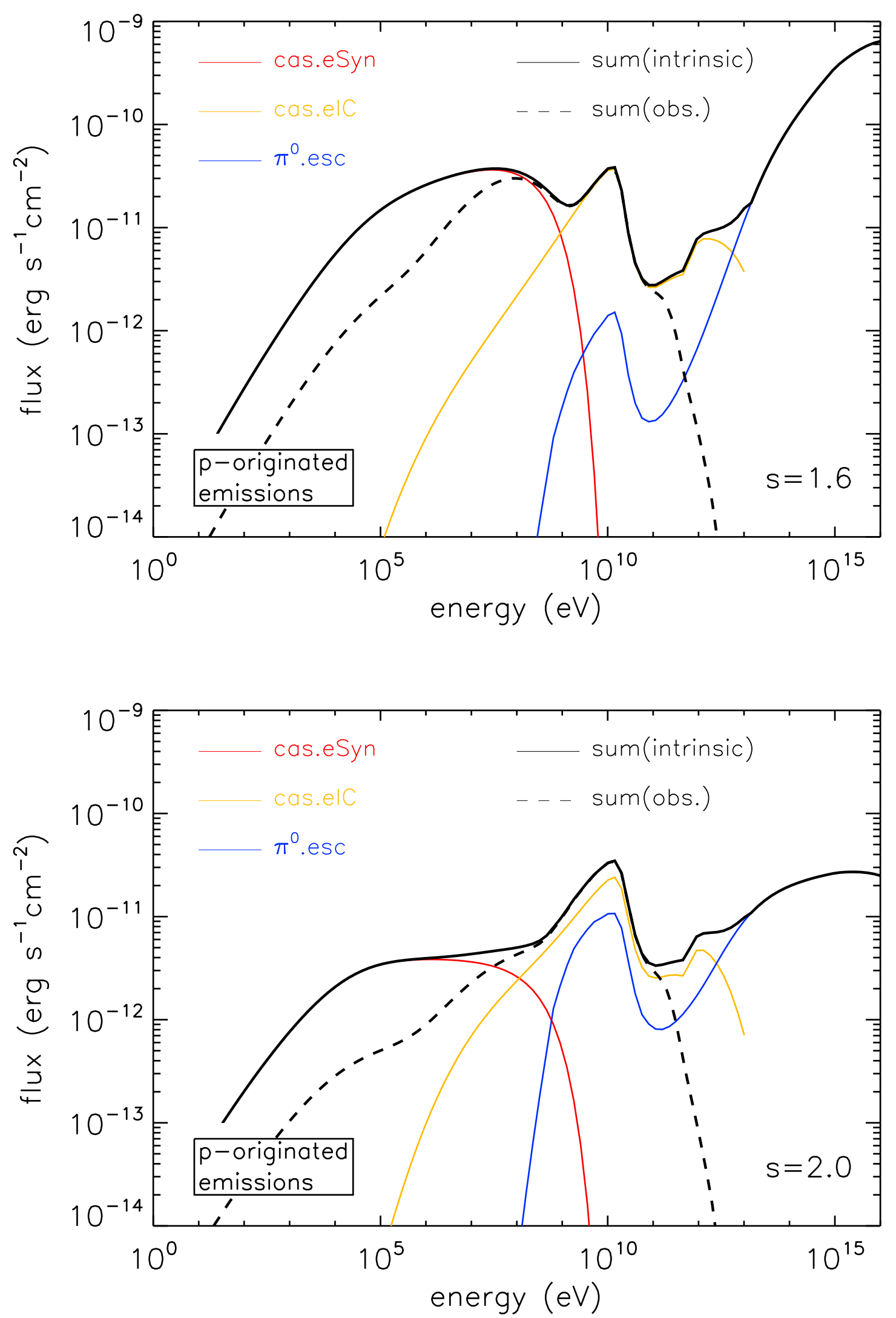

FIG. 5. Fluxes of various hadronic-originated emissions in the BLR. The red and orange solid curves represent, respectively, the synchrotron and inverse Compton radiation of electrons generated in the cascade. The blue curve represents escaping pionic gamma rays (i.e., gamma rays that are not annihilated and hence do not attend the cascade process). The black solid curve are the summon of the above three components and the black dashed curves are the flux after absorption through photoionisation and due to EBL absorption. The upper panel is for $s=1.6$ and the lower panel is for $s=2.0$. 
Assuming the clouds are composed of pure hydrogens, the photoionisation cross section can be given by [49]

$$
\sigma_{\mathrm{PI}}=\left\{\begin{array}{l}
\sigma_{0}\left(\frac{E_{\gamma}}{I_{H}}\right)^{-3}, \text { for } I_{H}<E_{\gamma} \lesssim 100 I_{H}, \\
\frac{3 e^{4}}{2 \pi} \sigma_{0}\left(\frac{E_{\gamma}}{I_{H}}\right)^{-3.5}, \text { for } E_{\gamma}>100 I_{H}
\end{array}\right.
$$

where $I_{H}=13.6 \mathrm{eV}$ is the ionisation energy of atomic hydrogen, $\sigma_{0}=6.3 \times 10^{-18} \mathrm{~cm}^{2}$ is the cross section at threshold and $e \simeq 2.72$ is the Euler's number. Take $s=2.0$ case as example, we calculate the the photoionisation rate by

$$
\zeta_{\text {ion }}=\int_{I_{H}}^{\infty} n_{\gamma}\left(E_{\gamma}\right) \sigma_{\mathrm{PI}} c d E_{\gamma} \simeq 100 \mathrm{~s}^{-1}
$$

where $n_{\gamma}\left(E_{\gamma}\right)$ is the differential photon number density based on the unabsorbed flux emitted by the BLR shown in Fig. 5. The recombination rate of pure hydrogen gas is given by $\zeta_{\text {rec }}=3 \times 10^{-6} T^{-1 / 2} n_{H} \simeq$ $6\left(k T_{\mathrm{BLR}} / 1.9 \mathrm{eV}\right)^{-1 / 2}\left(\mathrm{n}_{\mathrm{H}} / 3 \times 10^{8}\right) \mathrm{s}^{-1}$. Thus, the BLR clouds that entered into the jet will be fully ionised. The $\mathrm{UV} / \mathrm{X}$-ray emission $s=1.6$ is stronger so we will reach the same conclusion. Note that if the metallicity of the cloud is not zero, the recombination rate will be further reduced. In addition to photoionisation, some clouds may directly interact with the jet and a shock may be driven in the cloud, and the cloud may also be ionised in this process. Ionised electrons will scatter the photons to other direction from our line of sight. The optical depth is $\tau_{\mathrm{sc}}=\sigma_{\mathrm{sc}} n_{H} R_{\mathrm{BLR}} \simeq 2$ for X-ray photons and we multiply a factor of $\exp \left(-\tau_{\mathrm{sc}}\right)$ to the obtained flux in the BLR region. Note that the Klein-Nishina effect will reduce the cross section for gamma rays, i.e. [50],

$$
\sigma_{\mathrm{sc}}=\sigma_{\mathrm{T}} \cdot \frac{3}{4}\left[\frac{1+x}{x^{3}}\left\{\frac{2 x(1+x)}{1+2 x}-\ln (1+2 x)\right\}+\frac{1}{2 x} \ln (1+2 x)-\frac{1+3 x}{(1+2 x)^{2}}\right],
$$

where $x=E_{\gamma} / m_{e} c^{2}$.

\section{Jet-cloud interactions:}

Neither the jet nor the BLR gas are homogeneously distributed in reality, but some BLR clouds may encounter with the jet material.The jet will exert a pressure on the cloud to accelerate the cloud along the jet propagation axis. Hydrodynamical instabilities can also occur leading to the cloud fragmentation [26]. Eventually, a considerable fraction of the cloud material may be loaded in the jet and jet is slowed down. Consider the SMBH launch a baryonic jet with kinetic luminosity equal to the Eddington luminosity, say, $L_{k}=1.3 \times 10^{47} \mathrm{erg} / \mathrm{s}$ for a SMBH mass of $10^{9} M_{\odot}$. We can estimate the mass of the segment of the jet from the jet base to the BLR is $\simeq L_{k} d_{\mathrm{BLR}} / \Gamma c^{3}=0.004 M_{\odot}$. Assume a transverse radius of the jet to be $r_{j}=10^{15} \mathrm{~cm}$, we can obtain the total mass of the BLR gas inside the jet to be $\pi r_{j}^{2} N_{H} \simeq 0.008 M_{\odot}$. Even if considering the extreme case, where all the BLR gas inside the jet are loaded in the jet, we expect the bulk Lorentz factor of the jet decrease to $\Gamma / 3$ considering conservation of kinetic energy and neglecting other kind of energy loss. For $\Gamma=20$ and a viewing angle of $4^{\circ}$ as employed in the calculation, the Doppler factor of the jet decrease from $\delta_{D}=13.6$ to $\delta_{D}=10.9$ after passing through the BLR. We do not expect slowdown of the jet will influence the leptonic emission of the blob outside the BLR significantly.

\section{Influence of an infrared photon field from dusty torus:}

Now let us study the effect of an additional infrared photon field supplied by the possibly existed dusty torus. The dusty torus generally locate at an extended region of $0.1-10 \mathrm{pc}$. Similar to the BLR, the torus absorbs part of the AGN emission and reprocesses it into infrared emissions, which consist of multiple grey body components of temperature ranging from $\sim 50 \mathrm{~K}$ to $1000 \mathrm{~K}$. High-energy gamma rays that escape the BLR may interact with the infrared photon field of the dusty torus, generate electron pairs and re-emit at lower energy. Let us consider that the dust of temperature $T_{\mathrm{DT}}$ emit at a luminosity of $L_{\mathrm{DT}}$, extending a spatial scale of $R_{\mathrm{DT}}$. They supply a photon field of number density

$$
n_{\mathrm{ph}, \mathrm{DT}} \sim \frac{L_{\mathrm{IR}}}{3 k T_{\mathrm{DT}} \pi R_{\mathrm{DT}}^{2} c} \simeq 8 \times 10^{5}\left(L_{\mathrm{IR}} / 10^{41} \mathrm{erg} \mathrm{s}^{-1}\right)\left(R_{\mathrm{DT}} / 1 \mathrm{pc}\right)^{-2}\left(T_{\mathrm{DT}} / 300 \mathrm{~K}\right)^{-1} \mathrm{~cm}^{-3}
$$

within a scale of $R_{\mathrm{DT}}$ around the SMBH. For hot dust of temperature $1000 \mathrm{~K}$ extending a spatial scale of $R_{\mathrm{DT}}=0.1 \mathrm{pc}$, we obtain a photon number density of $n_{\mathrm{ph}, \mathrm{DT}}=3 \times 10^{7} \mathrm{~cm}^{-3}$ with $L_{\mathrm{DT}}=10^{41} \mathrm{erg} / \mathrm{s}$ which is comparable to the BLR luminosity. Such an infrared photon field typically absorbs $\sim 4 \mathrm{TeV}$ gamma rays. The optical depth of $\gamma \gamma$ annihilation can be estimated by $\tau_{\gamma \gamma, \mathrm{DT}} \simeq n_{\mathrm{ph}, \mathrm{DT}} \sigma_{\gamma \gamma} R_{\mathrm{DT}} \simeq 0.8$ for gamma-ray photons of energy $4 \mathrm{TeV}$ typically. The photon density from hot dust will drop quickly and become anisotropic at the region beyond $0.1 \mathrm{pc}$ and hence do not further contribute the optical depth. Similarly, we can obtain the optical depth by warm dust of $300 \mathrm{~K}$ at a scale of $1 \mathrm{pc}$ by $\tau_{\gamma \gamma, \mathrm{DT}} \simeq 0.2$ for $\sim 10 \mathrm{TeV}$ gamma rays and by cold dust of $50 \mathrm{~K}$ at a scale of $10 \mathrm{pc}$ by $\tau_{\gamma \gamma, \mathrm{DT}} \simeq 0.1$ for $\sim 80 \mathrm{TeV}$ gamma 
rays, if we assume the luminosity of each of these emitters is $L_{\mathrm{DT}}=10^{41} \mathrm{erg} / \mathrm{s}$. Therefore, only a small fraction of the energy of escaping gamma rays will go into lower energy emission and do not add to the jet emission.

Assuming the infrared photon field is composed of grey body emissions of the dusty torus of three temperatures at different spatial scales, we employ Eqs.(3)-(8) to deal with the cascade emission in the infrared photon field with a few modifications: (i) for the electron injection, the term $Q_{e, \pi}^{\prime}$ will not show up in Eq. (3) since there is no target for $p p$ collision beyond the BLR; (ii) Eq. (5) now reads

$$
\begin{aligned}
Q_{e, \gamma \gamma}^{\mathrm{DT}}\left(\gamma_{e}^{\prime}\right)^{\prime} & =f_{\mathrm{abs}}^{\mathrm{DT}}\left(E_{\gamma, 1}^{\prime}\right)\left(\dot{n}_{E_{\gamma, 1}^{\prime} \mathrm{sy}}^{\prime}+\dot{n}_{E_{\gamma, 1}^{\prime}}^{\mathrm{IC}}\right)+g_{\mathrm{abs}}^{\mathrm{DT}} \dot{n}_{E_{\gamma, 1}^{\prime}}^{\mathrm{BLR}} \\
& +f_{\mathrm{abs}}^{\mathrm{DT}}\left(E_{\gamma, 2}^{\prime}\right)\left(\dot{n}_{E_{\gamma, 2}^{\prime} \mathrm{sy}}^{\mathrm{s}}+\dot{n}_{E_{\gamma, 2}^{\prime}}^{\mathrm{IC}}\right)+g_{\mathrm{abs}}^{\mathrm{DT}} \dot{n}_{E_{\gamma, 2}^{\prime}}^{\mathrm{BLR}},
\end{aligned}
$$

where $\dot{n}_{E_{\gamma, 1 / 2}^{\prime}}^{\mathrm{BLR}}$ is the photon emission rate of the BLR obtained above. $f_{\mathrm{abs}}^{\mathrm{DT}}$ holds the same form of Eq. 6] while $g_{\mathrm{abs}}^{\mathrm{DT}}=1-e^{-\tau_{\gamma \gamma, D T}}$ because photons injected from the BLR will penetrate the whole infrared photon field; (iii) for cascade emission in the infrared photon field, there is no opacity from ionised electrons since the region where the cascade develops is far beyond the BLR of high column density. We compare photon fluxes obtained with and without considering the emission of dusty torus in Fig. 6. As can be seen, the predicted flux almost does not change after introducing the infrared emission from the dusty torus. The magnetic field in this extended region of of $R=0.1-10 \mathrm{pc}$ is supposed to be much weaker than that in the BLR since this region is far beyond the dissipation region. We the magnetic field strength decrease as $R^{-2}$ (i.e., magnetic flux conserves) in the calculation. At a larger spatial scale of $\sim 100 \mathrm{pc}$, the escaping gamma rays can be absorbed by the reprocessed emissions of dust in the starburst region of the host galaxy. We assume the generated electrons will be isotropised and their emission is negligible compared to the jet emission.

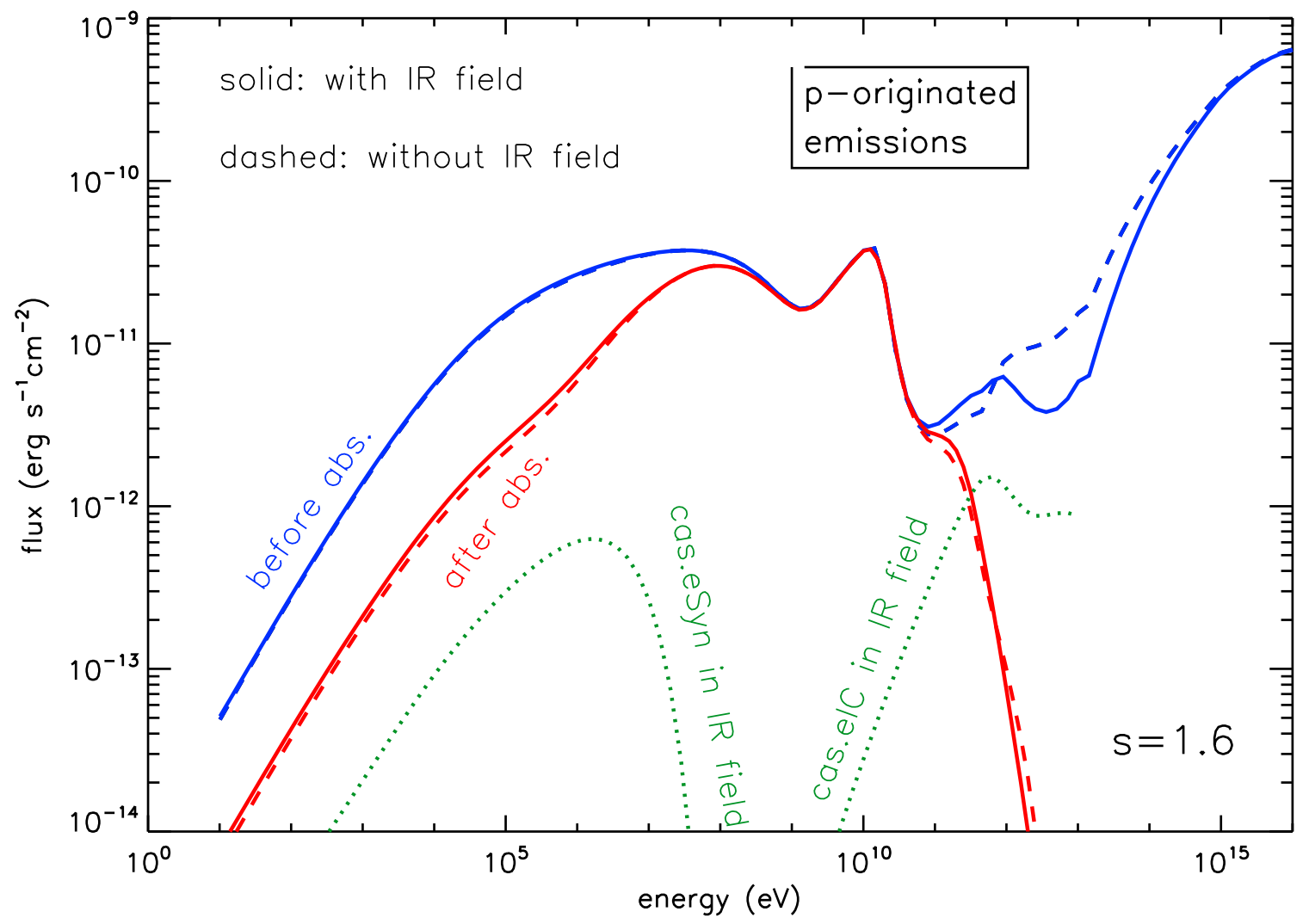

FIG. 6. Comparison of hadronic-originated fluxes between the case with (solid curves) considering the infrared photon field emitted by the dusty torus and the case without (dashed curves) considering it. The red curves represent the fluxes after the attenuation due to EBL (at high-energy end) and due to Compton scattering of the electrons in the BLR (at low-energy end), while the blue curves represent the flux before the attenuation. The green dotted curve shows the synchrotron radiation and $\mathrm{IC}$ radiation of electrons generated in the infrared photon field. 


\section{Leptonic emission of the blob:}

We assume relativistic electrons are injected in the blob with a luminosity $L_{e}^{\text {blob }}$ measured in the source frame. To reproduce the emission in optical to soft X-ray band, we employ a broken power-law function for the electron injection spectrum, with a broken energy $E_{e, b}^{\prime}$ and spectral index $s_{1}$ and $s_{2}$ below and above the break, respectively, i.e.,

$$
\dot{N}_{e, \mathrm{blob}}^{\prime} \propto \begin{cases}\left(\frac{E_{e}^{\prime}}{E_{e, b}^{\prime}}\right)^{-s_{1}}, & E_{e, 0}^{\prime} \leq E_{e}^{\prime}<E_{e, b}^{\prime} \\ \left(\frac{E_{e}^{\prime}}{E_{e, b}^{\prime}}\right)^{-s_{2}}, & E_{e}^{\prime} \geq E_{e, b}^{\prime}\end{cases}
$$

with $E_{e, 0}^{\prime}$ being the minimum energy of the injected electron. Similar to the case of protons in the BLR, we can obtain the normalisation of the electron injection spectrum by $\int E_{e}^{\prime} \dot{N}_{e, \text { blob }}^{\prime} d E_{e}^{\prime}=L_{e, \text { blob }} / \Gamma_{j}^{2}$. The total electron spectrum in the blob comoving frame is then $N_{e, \text { blob }}^{\prime}=\dot{N}_{e, \text { blob }}^{\prime} t_{e}^{\prime}$ where $t_{e}^{\prime}=\min \left(t_{c}^{\mathrm{blob}^{\prime}}, t_{\mathrm{esc}}^{\mathrm{blob}^{\prime}}, t_{\mathrm{dyn}}^{\mathrm{blob}^{\prime}}\right)$, representing the electron cooling timescale, the escape timescale and the dynamical timescale of the blob respectively. In the blob, the electron cooling is due to the synchrotron radiation and the synchrotron self-Compton scattering, so we have $t_{c}^{\text {blob }^{\prime}}=\frac{3 m_{e}^{2} c^{3}}{4 \sigma_{T}} \frac{E_{e}^{\prime}}{u_{B}^{\text {blob }}+u_{\text {syn }}^{\prime}}$ where $u_{B}^{\text {blob }}$ and $u_{\text {syn }}^{\prime}$ is the energy density of the magnetic field and the energy density of synchrotron radiation of the electrons in the blob in the blob. To calculate $u_{\text {syn }}^{\prime}$, we firstly get the luminosity of the synchrotron radiation by the electrons $L_{\mathrm{syn}}^{\prime}$, and then we can find $u_{\mathrm{syn}}^{\prime}$ by $u_{\mathrm{syn}}^{\prime}=L_{\mathrm{syn}} / \pi R_{\mathrm{blob}}^{2} c$. Usually, $t_{\mathrm{esc}}^{\mathrm{blob}^{\prime}}=R_{\mathrm{blob}}^{2} / D_{\mathrm{blob}}$ and $t_{\mathrm{dyn}}^{\prime}=d_{\mathrm{blob}} / \Gamma_{j} v_{j}$ are much larger than $t_{c}^{\prime}$.

TABLE I. Main parameters used in the spectral fittings.

\begin{tabular}{|c|c|c|c|}
\hline Parameters & Descriptions & \multicolumn{2}{|c|}{ Values } \\
\hline$s$ & proton spectral index at injection & $s=1.6$ & $s=2.0$ \\
\hline$L_{p}^{\mathrm{BLR}}$ & luminosity of protons injected in the BLR & $3.2 \times 10^{46} \mathrm{erg} \mathrm{s}^{-1}$ & $4.8 \times 10^{45} \mathrm{erg} \mathrm{s}^{-1}$ \\
\hline$L_{\mathrm{BLR}}$ & luminosity of the grey body emission of the BLR & $6.4 \times 10^{41} \mathrm{erg} \mathrm{s}^{-1}$ & $3.2 \times 10^{41} \mathrm{erg} \mathrm{s}^{-1}$ \\
\hline$c_{\mathrm{BLR}}$ & dilution factor of the grey body emission & $1.2 \times 10^{-4}$ & $6 \times 10^{-5}$ \\
\hline$B_{\mathrm{BLR}}^{\prime}$ & magnetic field of the BLR in the jet comoving frame & \multicolumn{2}{|c|}{$0.2 \mathrm{G}$} \\
\hline$\eta$ & the ratio of the mean free path to Larmor radius of protons in the BLR & \multicolumn{2}{|c|}{10} \\
\hline$T_{\mathrm{BLR}}$ & temperature of the grey body emission of the BLR & \multicolumn{2}{|c|}{$1.9 \mathrm{eV}$} \\
\hline$d_{\mathrm{BLR}}$ & mean distance of the BLR to the SMBH & \multicolumn{2}{|c|}{$3 \times 10^{16} \mathrm{~cm}$} \\
\hline$R_{\mathrm{BLR}}$ & size of the BLR & \multicolumn{2}{|c|}{$10^{16} \mathrm{~cm}$} \\
\hline$N_{\mathrm{H}}$ & column density of the BLR & \multicolumn{2}{|c|}{$10^{24.5} \mathrm{~cm}^{-2}$} \\
\hline$n_{\mathrm{H}}$ & mean atomic density of the BLR & \multicolumn{2}{|c|}{$3 \times 10^{8} \mathrm{~cm}^{-3}$} \\
\hline$\Gamma_{j}$ & bulk Lorentz factor of the jet(blob) ${ }^{a}$ & \multicolumn{2}{|c|}{20} \\
\hline$\beta_{j}$ & bulk speed of the jet(blob) in unit of $c$ & \multicolumn{2}{|c|}{0.9987} \\
\hline$\theta$ & viewing angle of the jet(blob) & \multicolumn{2}{|c|}{$4^{\circ}$} \\
\hline$\delta_{D}$ & Doppler factor of the jet(blob) ${ }^{b}$ & \multicolumn{2}{|c|}{13.6} \\
\hline$d_{\text {blob }}$ & distance of the blob to the SMBH & \multicolumn{2}{|c|}{$3 \times 10^{18} \mathrm{~cm}$} \\
\hline$R_{\text {blob }}$ & size of the blob & $10^{16.86} \mathrm{~cm}$ & $10^{16.95} \mathrm{~cm}$ \\
\hline$B_{\text {blob }}^{\prime}$ & magnetic field of the BLR in the blob comoving frame & $0.32 \mathrm{G}$ & $0.21 \mathrm{G}$ \\
\hline$L_{e}^{\text {blob }}$ & luminosity of electrons injected in the blob & $9.3 \times 10^{44} \mathrm{erg} \mathrm{s}^{-1}$ & $1.5 \times 10^{45} \mathrm{erg} \mathrm{s}^{-1}$ \\
\hline$E_{e, b}^{\prime}$ & break energy in the electron spectrum injected to the blob & $14.1 \mathrm{GeV}$ & $7.2 \mathrm{GeV}$ \\
\hline$E_{e, 0}^{\prime}$ & minimum energy of the electron injected to the blob & $0.005 \mathrm{GeV}$ & $0.005 \mathrm{GeV}$ \\
\hline$s_{1}$ & electron spectral index before the break & 1.67 & 1.72 \\
\hline$s_{2}$ & electron spectral index after the break & 4.63 & 3.78 \\
\hline
\end{tabular}

$a$ : the bulk Lorentz factors of the blobs inside the BLR and outside the BLR are not necessarily the same.

$b: \delta_{D}=\left[\Gamma_{j}\left(1-\beta_{j} \cos \theta\right)\right]^{-1}$.

After we obtain the differential luminosity of both the emissions from the BLR and the blob in the comoving frame, i.e., $L^{\prime}\left(E_{\gamma}^{\prime}\right)=L_{\mathrm{BLR}}^{\prime}+L_{\mathrm{blob}}^{\prime}$, we can calculate the flux at the Earth by

$$
f_{\gamma}\left(E_{\gamma}\right)=\frac{\Gamma_{j}^{2} \delta_{D}^{2} L^{\prime}\left(E_{\gamma}^{\prime}\right)}{4 \pi D_{L}^{2}} e^{-\tau_{\gamma \gamma}^{\mathrm{EBL}}\left(E_{\gamma}, z\right)} \frac{d E_{\gamma}^{\prime}}{d E_{\gamma}}
$$

where the factor $\Gamma_{j}^{2}$ accounts for the beaming effect due to relativistic motion of the jet (blob) while $\delta_{D}^{2}$ considers the Doppler boost of the flux. $D_{L}=1.77 \mathrm{Gpc}$ is the luminosity distance for the redshift $z=0.3365$, while $d E_{\gamma}=$ 
$\delta_{D} d E_{\gamma}^{\prime} /(1+z) \cdot \tau_{\gamma \gamma}^{\mathrm{EBL}}$ is the optical depth for gamma-ray photons due to the absorption by the extragalactic background light (EBL). Here we employ the EBL model provided by [51]. Note that a pure leptonic model can give an acceptable fitting to the multiwavelength flux. The hadronic process is considered mainly for the neutrino production.

[1] IceCube Collaboration, Science 342, 1242856 (2013) arXiv:1311.5238 [astro-ph.HE]

[2] R.-Y. Liu, X.-Y. Wang, S. Inoue, R. Crocker, and F. Aharonian, Physical Review D 89, 083004 (2014) arXiv:1310.1263 [astro-ph.HE]

[3] I. Tamborra, S. Ando, and K. Murase, JCAP 9, 043 (2014), arXiv:1404.1189 [astro-ph.HE]

[4] X.-C. Chang, R.-Y. Liu, and X.-Y. Wang, The Astrophysical Journal 805, 95 (2015) arXiv:1412.8361 [astro-ph.HE]

[5] X.-Y. Wang and R.-Y. Liu, Physical Review D 93, 083005 (2016) arXiv:1512.08596 [astro-ph.HE]

[6] C. Lunardini and W. Winter, Physical Review D 95, 123001 (2017). arXiv:1612.03160 [astro-ph.HE]

[7] N. Senno, K. Murase, and P. Mészáros, The Astrophysical Journal 838, 3 (2017) arXiv:1612.00918 [astro-ph.HE]

[8] F. W. Stecker, Physical Review D 88, 047301 (2013). arXiv:1305.7404 [astro-ph.HE]

[9] K. Murase, Y. Inoue, and C. D. Dermer, $\quad$ Physical Review D 90, 023007 (2014), arXiv:1403.4089 [astro-ph.HE]

[10] F. Tavecchio and G. Ghisellini, Mon. Not. R. Astron. Soc. 451, 1502 (2015), arXiv:1411.2783 [astro-ph.HE]

[11] M. Petropoulou, S. Dimitrakoudis, P. Padovani, A. Mastichiadis, and E. Resconi, Mon. Not. R. Astron. Soc. 448, 2412 (2015), arXiv:1501.07115 [astro-ph.HE]

[12] P. Padovani, M. Petropoulou, P. Giommi, and E. Resconi, Mon. Not. R. Astron. Soc. 452, 1877 (2015), arXiv:1506.09135 [astro-ph.HE]

[13] C. Kopper and E. Blaufuss, GRB Coordinates Network, Circular Service, No. 21916, \#1 (2017) 21916 (2017).

[14] The IceCube, Fermi-LAT, MAGIC, AGILE, ASAS-SN, HAWC, H.E.S.S., INTEGRAL, Kanata, Kiso, Kapteyn, Liverpool telescope, Subaru, Swift/NuSTAR, VERITAS, and VLA/17B-403 teams, Science 361 (2018), 10.1126/science.aat1378, http://science.sciencemag.org/content/361/6398/eaat1378.

[15] S. Paiano, R. Falomo, A. Treves, and R. Scarpa, The Astrophysical Journal Letter 854, L32 (2018), arXiv:1802.01939.

[16] Y. T. Tanaka, S. Buson, and D. Kocevski, The Astronomer's Telegram 10791 (2017).

[17] R. Mirzoyan, The Astronomer's Telegram 10817 (2017).

[18] D. B. Fox, J. J. DeLaunay, A. Keivani, P. A. Evans, C. F. Turley, J. A. Kennea, D. F. Cowen, J. P. Osborne, M. Santander, and F. E. Marshall, The Astronomer's Telegram 10845 (2017).

[19] A. Franckowiak, K. Z. Stanek, C. S. Kochanek, T. A.
Thompson, T. W.-S. Holoien, B. J. Shappee, J. L. Prieto, and S. Dong, The Astronomer's Telegram 10794 (2017); T. Morokuma, Y. T. Tanaka, K. Ohta, Y. Matsuoka, T. Yamashita, and N. Kato, ibid. 10890 (2017); M. Yamanaka, Y. T. Tanaka, H. Mori, K. S. Kawabata, Y. Utsumi, T. Nakaoka, M. Kawabata, and H. Nagashima, ibid. 10844 (2017); A. Coleiro and S. Chaty, ibid. 10840 (2017).

[20] A. J. Tetarenko, G. R. Sivakoff, A. E. Kimball, and J. C. A. Miller-Jones, The Astronomer's Telegram 10861 (2017).

[21] A.-C. Donea and R. J. Protheroe, Astroparticle Physics 18, 377 (2003), astro-ph/0202068

[22] R. Morganti, M.-H. Ulrich, and C. N. Tadhunter, Mon. Not. R. Astron. Soc. 254, 546 (1992).

[23] R. C. Vermeulen, P. M. Ogle, H. D. Tran, I. W. A. Browne, M. H. Cohen, A. C. S. Readhead, G. B. Taylor, and R. W. Goodrich, The Astrophysical Journal Letter 452, L5 (1995)

[24] E. A. Corbett, A. Robinson, D. J. Axon, and J. H. Hough, Mon. Not. R. Astron. Soc. 311, 485 (2000)

[25] A. Dar and A. Laor, The Astrophysical Journal Letter 478, L5 (1997) astro-ph/9610252

[26] A. T. Araudo, V. Bosch-Ramon, and G. E. Romero, Astronomy and Astrophysics 522, A97 (2010), arXiv:1007.2199 [astro-ph.HE]

[27] B. M. Peterson, in Physics of Active Galactic Nuclei at all Scales, Lecture Notes in Physics, Berlin Springer Verlag, Vol. 693, edited by D. Alloin (2006) p. 77.

[28] H. Netzer, Annu. Rev. Astron. Astrophys 53, 365 (2015) arXiv:1505.00811.

[29] J. A. Baldwin, G. J. Ferland, K. T. Korista, F. Hamann, and M. Dietrich, The Astrophysical Journal 582, 590 (2003), astro-ph/0209335

[30] B. Zhang and H. Yan, The Astrophysical Journal 726, 90 (2011) arXiv:1011.1197 [astro-ph.HE]

[31] J. Stern and A. Laor, Mon. Not. R. Astron. Soc. 426, 2703 (2012), arXiv:1207.5543

BB]dF. Murase, D. Guetta, and M. Ahlers, Physical Review Letters 116, 071101 (2016) arXiv:1509.00805 [astro-ph.HE]

[33] S. R. Kelner, F. A. Aharonian, and V. V. Bugayov, Physical Review D 74, 034018 (2006) astro-ph/0606058

[34] M. Böttcher, A. Reimer, K. Sweeney, and A. Prakash, The Astrophysical Journal 768, 54 (2013) arXiv:1304.0605 [astro-ph.HE]

[35] K. Wang, R.-Y. Liu, Z.-G. Dai, and K. Asano, The Astrophysical Journal 857, 24 (2018) arXiv:1803.04112 [astro-ph.HE] 
[36] S. Inoue and F. The Astrophysical Journal 463, 555 (1996)

[37] F. Tavecchio, L. Maraschi, and G. Ghisellini, The Astrophysical Journal 509, 608 (1998) astro-ph/9809051.

[38] L. Costamante and G. Ghisellini, Astronomy and Astrophysics 384, 56 (2002), astro-ph/0112201.

[39] S. Gao, M. Pohl, and W. Winter, The Astrophysical Journal 843, 109 (2017), arXiv:1610.05306 [astro-ph.HE]

[40] F. W. Stecker, C. Done, M. H. Salamon, and P. Sommers, Physical Review Letters 66, 2697 (1991)

[41] K. Mannheim, Physical Review D 48, 2408 (1993), astro-ph/9306005

[42] A. Atoyan and C. D. Dermer, Physical Review Letters 87, 221102 (2001). astro-ph/0108053.

[43] A. Mücke, R. J. Protheroe, R. Engel, J. P. Rachen, and T. Stanev, Astroparticle Physics 18, 593 (2003), astro-ph/0206164

[44] IceCube Collaboration, Science 361, 147 (2018)

[45] F. W. Stecker, Astrophysics and Space Science 6, 377 (1970)

[46] E. Kafexhiu, F. Aharonian, A. M. Taylor, and G. S. Vila, Physical Review D 90, 123014 (2014), arXiv:1406.7369 [astro-ph.HE]

[47] V. L. Ginzburg and S. I. Syrovatskii, The Origin of Cosmic Rays, New York: Macmillan, 1964 (1964).

[48] F. A. Aharonian, A. M. Atoyan, and A. M. Nagapetyan, Astrophysics 19, 187 (1983)

[49] B. T. Draine, Physics of the Interstellar and Intergalactic Medium by Bruce T. Draine. Princeton University Press, 2011. ISBN: 978-0-691-12214-4 (2011).

[50] G. B. Rybicki and A. P. Lightman, Radiative Processes in Astrophysics, by George B. Rybicki, Alan P. Lightman, pp. 400. ISBN 0-471-82759-2. Wiley-VCH , June 1986. (1986) p. 400.

[51] J. D. Finke, S. Razzaque, and C. D. Dermer, The Astrophysical Journal 712, 238 (2010), arXiv:0905.1115 [astro-ph.HE] 